\title{
The Tolerance and Safety of 4-Demethyl-4- cholesteryloxypenclomedine [DM-CHOC-PEN] in Adolescent and Young Adult (AYA) Subjects with Advanced Malignancies
}

\author{
Weiner RS ${ }^{1}$, Ware $\mathrm{ML}^{1,2}$, Bhandari $\mathrm{M}^{3}$, Mahmood $\mathrm{T}^{4}$, Friedlander $\mathrm{P}^{5}$ and Morgan $\mathrm{LR}^{1,6 *}$ \\ ${ }^{1}$ Tulane University Medical Center, New Orleans, LA, USA \\ ${ }^{2}$ Ochsner Medical Center, New Orleans, LA, USA \\ ${ }^{3}$ The Christ Hospital, Cincinnati, OH, USA \\ ${ }^{4}$ Detroit Clinical Research Centers, Detroit, Owosso \& Lansing, MI, USA \\ ${ }^{5}$ Mount Sinai Medical Center, New York, NY, USA \\ ${ }^{6}$ DEKK-TEC, Inc. New Orleans, LA, USA
}

\section{Introduction}

Almost 700,000 people in the US are living with tumors involving the central (CNS) or spinal nervous system (SNS) tumors (1). Nearly $15 \%$ of these tumors occur in adolescents or young adults (AYA), aged 15-39 years of age (2). It is predicted that 10,617 AYA individuals will be diagnosed with brain or CNS tumors resulting in 434 deaths this year in the US [1]. AYA individuals with histories of cancer, who appeared to have 'beaten the odds' are now developing recurrent cancers involving the CNS years after remission in increasing rates since 1989 [2,3]. The most common types of cancer in AYA individuals are primary brain tumors (glioblastoma (GBM), etc.) and metastatic cancers - melanoma, leukemia and sarcomas involving the CNS [2]. AYA groups with cancer possess different host biology, tumor pathophysiology and metabolize drugs differently than do either younger or older individuals that may have the chronic disease histories and may respond differently to chemotherapy [4-6].

For male and female individuals $<20$ years of age, primary brain and secondary cancers of the CNS and (SNS) are the most common causes of death from cancer; in the 20-39 year age group, they are the first cause of cancer-related deaths in males and the fifth cause of cancer-related deaths in females [1]. The incidence and histology of cancer types varies according to patient age [1]. Tumors in these age groups warrant effective innovative treatment.

\section{Innovation and significance}

4-Demethyl-4-cholesteryloxypenclomedine [DM-CHOC-PEN] (Figure 1) is a polychlorinated pyridine cholesteryl carbonate designed and developed at DEKK-TEC that is lipophilic, electrically neutral and able to cross the blood brain barrier (BBB) [7-9]. The drug has been evaluated in Phase I/II clinical trials with objective responses and has improved over-all survival and PFS in subjects with advanced cancer involving the CNS and SNS (Table 1) (IND 68,876; NCT02889445) $[9,10]$.

A total of fifty three (53) adult subjects with advanced cancers have been treated to date in clinical trials with DM-CHOC-PEN; forty-five subjects had CNS involvement (IND 68,876) $[8,9]$. The most common types of cancers treated have been metastatic lung, breast, melanoma, and primary brain (GBM, astrocytoma) cancers involving the CNS [10].

In adults, the most common adverse effects associated with DMCHOC-PEN were fatigue, liver dysfunction $(\mathrm{Gr}-3, \mathrm{n}=3)$ and nausea $(\mathrm{Gr}-2, \mathrm{n}=2)$; all of which were transient. Hematology, renal, cardiac, pulmonary and neurological/cognitive profiles remained stable throughout the trials [8-10]. There have been no toxicities in the AYA aged group to date; however, no AYA subject treated has had preexisting liver or other chronic non-malignant illnesses.

To date, seven subjects have been treated in the AYA age group; all had cancer involving the CNS (Figure 2).

Table 1 reviews these AYA subjects, their doses (39, 55, or 97.8 $\mathrm{mg} / \mathrm{m}^{2}$ administered once every 21 days), the responses and toxicities. Several subjects were treated at more than 1-dose level. The AYA subjects had advanced, chemo-resistant stage IV cancer - melanoma, NSCLC, breast, acute lymphocytic leukemia, oligodendroglioma and astrocytoma $[9,10]$.<smiles>[R]Oc1c(Cl)c(OC)nc(C(Cl)(Cl)Cl)c1Cl</smiles>

Figure 1. Penclomedine analogs $-\mathrm{PEN}\left(\mathrm{R}=\mathrm{CH}_{3}\right)$; DM-PEN $(\mathrm{R}=\mathrm{H})$; DM-CHOC-PEN $(\mathrm{R}=$ $\mathrm{CO}_{2}$-cholesteryl)

Correspondence to: Lee Roy Morgan, Tulane University Medical Center, New Orleans, LA, USA, Tel: 504-583-6135; E-mail: Irml1579@aol.com

Received: May 22, 2017; Accepted: June 13, 2017; Published: June 15, 2017 


\section{PROPOSED MECHANISM OF ACTION OF 4-DEMETHYL- 4-CHOLESTEROLYL-PENCLOMEDINE (DM-CHOC-PEN)}<smiles>COc1nc(C(Cl)(Cl)Cl)c(Cl)c(OC=O)c1Cl</smiles><smiles>COC1=NC(Cl)=C(Cl)C(=O)C1C</smiles>

$$
\begin{array}{l|l}
-\mathrm{HCl} & \begin{array}{l}
\text { DNA } \\
\text { interstrand } \\
\text { crosslinking }
\end{array}
\end{array}
$$<smiles>CC(C)[C@H]1C(=O)C(Cl)=C(Cl)N=C1Cl</smiles>

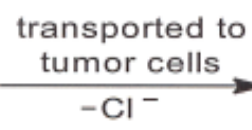

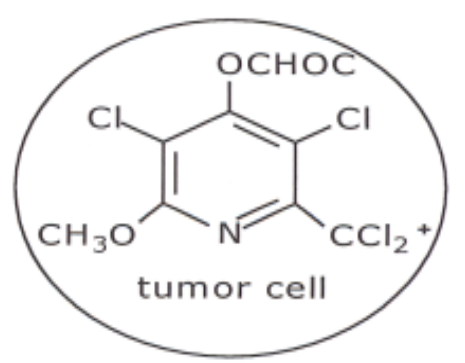

carbonylated carbonium ion

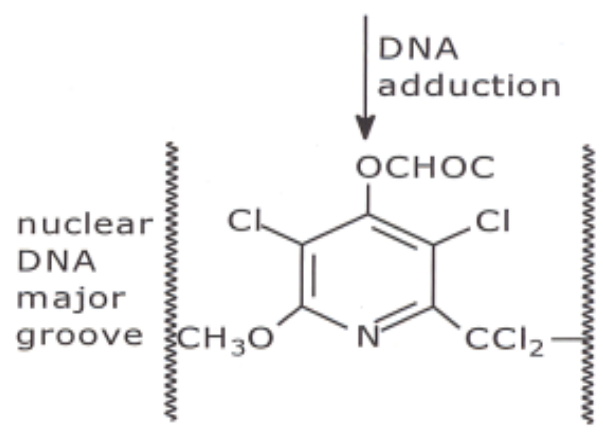

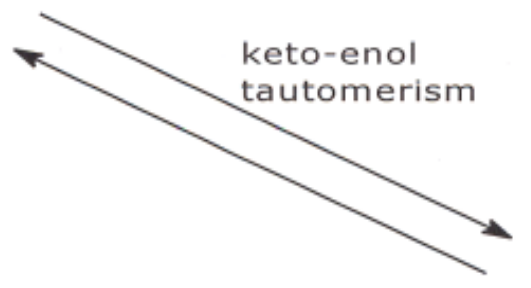

$$
\text { -CHOC-OH } \begin{aligned}
& \text { Tumor } \\
& \text { cell } \\
& \text { esterase }
\end{aligned}
$$<smiles>COc1nc(Cl)c(Cl)c(O)c1Cl</smiles>

\section{$\mathrm{CHOC}=\stackrel{\stackrel{\mathrm{O}}{\mathrm{C}}-\mathrm{O}-\text { cholesteryl }}{\mathrm{C}}$}

Figure 2. Proposed Mechanism of action of 4-Demethyl-4-Cholestrolyl-Penclomedine (DM-CHOC-PEN)

Unlike other penclomedines (e.g., PEN, NSC 338720, Figure 1), DM-CHOC-PEN is non-neurotoxic; however, it does cross the blood brain barrier $(\mathrm{BBB})$ in animals and humans with responses reported in sarcoma, astrocytoma, melanoma, ALL, lung, and breast cancers involving the spinal and central nervous systems in clinical trials [10]. The drug accumulated (and was quantitated - 61-120 ng/g of tumor tissue) in human sarcoma and lung cancer tissues involving the CNS/
SNS and was not found in adjacent normal brain/spinal tissue from subjects in Phase I/II trials [8-10].

DM-CHOC-PEN does not require hepatic activation and is active in nanogram quantities in vitro against melanoma GBM and breast cancer explants $[7,8]$. This is in contrast to DM-PEN (Figure 1) and other analogs that require hepatic activation [7]. These observations 
Weiner RS (2017) The Tolerance and Safety of 4-Demethyl-4-cholesteryloxypenclomedine [DM-CHOC-PEN] in Adolescent and Young Adult (AYA) Subjects with Advanced Malignancies

Table 1. AYA Subjects with Advanced Cancers Treated with IV DM-CHOC-PEN

\begin{tabular}{|c|c|c|c|c|c|}
\hline Cancer Type (\#) & Age/Sex & Dose $\left(\mathrm{mg} / \mathrm{m}^{2}\right)$ & Responders w/ CNS (\#) & OS (w/CNS) (mos) & Toxicity \\
\hline Melanoma (1) & $39 / \mathrm{F}$ & 98.7 & 0 & 2 & None \\
\hline NSCLC (1) & $39 / \mathrm{F}$ & 98.7 & 1 & $25+$ & None \\
\hline Breast (2) & $33 \& 29 / F$ & $39,55 \& 98.7$ & 1 & $12 \& 3$ & None \\
\hline Astrocytoma (1) & $37 / \mathrm{M}$ & 98.7 & 1 & $25+$ & None \\
\hline ALL (1) & $\mathrm{M} / 39$ & 98.7 & 1 & $8 * *$ & None \\
\hline NHL (1) & $\mathrm{F} / 28^{*}$ & 98.7 & NR & No CNS disease & None \\
\hline
\end{tabular}

*No CNS disease - no responses; $* * \mathrm{CNS}-\mathrm{CR}$, w/ peripheral progression

have led to a proposed mechanism whereby DM-CHOC-PEN associates with erythrocyte surface membranes, penetrates the BBB and brain parenchyma and is transported into intracerebral tumors with L-glutamine, with which it shares common structural moieties [11-13]. Thus, DM-CHOC-PEN may be killing micro-metastases, inhibiting DNA repair (an alkylating agent), and inducing an 'abscopal' immune effect [11].

A Phase I clinical trial with DM-CHOC-PEN in AYA subjects (1539 years old) with advanced malignancies (+/- CNS involvement) [IND $68,876]$ has been approved by the FDA and is in progress to validate the observations in Table 1. Moreover, subjects - 15-39 years old - are of major interest since they generally possess less or no co-morbidities.

Enrollment criteria for the AYA subjects in the clinical trial include a Zubrod PS score of $0-2$, adequate renal/hepatic/hematological functions, and stable mental/neurological systems. Subjects with liver metastasis are eligible if transaminases and bilirubin are $<1.5 \mathrm{x}$ ULN. All subjects requiring full-dose anticoagulation are excluded.

The current Phase I AYA protocol (DTI-024) has been approved by the FDA, CTEP, IRBs and permits enrollments of subjects at escalating doses of 50,75 , and $100 \mathrm{mg} / \mathrm{m}^{2}$ once every 21 days [13]. New subjects can be added to a higher dose level after one or more subjects receives 2 -cycles of the drug at a given dose level (to avoid accumulative toxicity). When one severe limiting toxicity (SLT) is observed the study will be expanded to 5-6 patients at that dose level. Two (2) SLTs at the same dose levels will define the MAD (maximum administered dose). At least 5 subjects will be treated at the dose level below the MAD, and, if safe, this level will be the maximum tolerated dose (MTD) and the starting dose for future AYA Phase II trials [14,15].

The clinical toxicity observed for DM-CHOC-PEN in the adult Phase I/II trials has been primarily hepatic - elevated bilirubin. Initial concerns from the rat study that elevations in lipid profiles could be a limiting problem have not been substantiated in man. Transient hypercholesteremia and hyperlipidemia due to the vehicle plus metabolism of DM-CHOC-PEN to DM-PEN and cholesterol have been seen in the Phase I trial [10]. The lipid profiles (cholesterol and triglycerides) for the subjects were erratic during the 3-h IV infusion period ( $2^{\circ}$ to the lipid emulsion vehicle and release of cholesterol), however, they returned to pre-treatment values after 24 hours. Triglycerides were the most significantly affected [9]. Hepatic imaging also failed to demonstrate hepatic cysts $2^{\circ}$ to DM-CHOC-PEN in humans that were seen in the rat study $[9,10]$.

In all the clinical trials, hematology and renal profiles have remained stable, plus, a rigorous mental examination performed before, during and after each dosing, and during subsequent weekly visits demonstrated no abnormalities $[9,10]$. The mental status exam was approved by the FDA, satisfied all IRBs, and will be continued in DTI-024 [15].
AYA subjects with cancer involving the CNS are encouraged to participate in the present trial with DM-CHOC-PEN. The objective responses and lack of toxicity seen in AYA subjects with cancer involving the CNS that have been treated with DM-CHOC-PEN to date encouraged the development of this AYA trial [Table 1] (DTI-0124) to verify the drug's usefulness in a wider age range of AYA subjects with cancer [15].

\section{Impression}

AYA subjects that lack histories of chronic illnesses and comorbidities (hypertension, pulmonary issues, etc.) and have no prior evidence of hepatic dysfunction (liver metastasis and/or illicit drug use, allergies, etc.) may tolerate the drug differently from older subjects, but they may still be at risk for toxicity from DM-CHOC-PEN. Plus, since this age group is not commonly being enrolled in clinical trials, survival information regarding AYA individuals are not available and even worse the subjects may be missing maximum care $[16,17]$.

\section{Acknowledgements}

This research was supported by the following grants NCI/SBIR grants - R43/44CA132257; R43CA203351, LACaTS - U54GM104940-01.

\section{References}

1. Siegel RL, Miller KD, Jemal A (2017) Cancer Statistics, 2017. CA Cancer J Clin 67 7-30. [Crossref]

2. Wilson E (2016) Brain tumors affect adolescents and young adults differently. HemOnc today.

3. Termini J, Neman J, Jandial R (2014) Role of the neural niche in brain metastatic Cancer Res 74: 4011- 4015. [Crossref]

4. Franklin ARK (2016) A Growing identity for adolescent and young adult oncology Oncology Times 38: 8-10.

5. Hayes-Lattin (2016) Integrating AYA oncology care into the worlds of pediatric and adult oncology care to improve cancer outcomes. The ASCO Post December 10, 82-83.

6. Pemmaraju N (2017) Adolescent \& young adults with chronic myeloid leukemia Oncology Times 39: 25-26.

7. Morgan LR, Struck RF, Waud WR, Jursic BS, Serota D, et al. (2009) Carbonate and carbamate derivatives of 4-demethylpenclomedine as novel anticancer Agents. Cancer Chemother Pharmacol 64: 618-623. [Crossref]

8. Morgan LR, Rodgers AH, Bastian G, Benes E, Waud WS, et al. (2014) Comparative preclinical pharmacology and toxicology for 4-demethyl-4cholestryloxylcarbonylpenclomedine (DM-CHOC-PEN) - a potential neuro-alkylating agent for glioblastoma (GBM) and metastatic cancers involving the central nervous system. In: Tumors of the Central Nervous System - Primary and Secondary, Rijeka InTech Pp: 239-263.

9. Weiner RS, Friedlander P, Gordon C, Saenger Y, Ware RL, et al. (2014) A phase I cancer clinical trial for 4-demethyl-4-cholesteryl-oxycarbonylpenclomedine (DMCHOC-PEN). Proc Am Assoc Cancer Res 56: 746.

10. Weiner RS, Friedlander P, Gordon C, Saenger Y, Ware RL, et al. (2016) Results of Phase II cancer clinical trials for 4-demethyl-4-cholesteryoxycarbonylpenclomedine (DM-CHOC-PEN). Proc Am Assoc Cancer Res 58: 236.

11. Morgan LR, Benes E, Rodgers AH, Weiner RS, Mahmood T (2015) Penetration of NSCLC involving the CNS via L-glutamine transport. 16th Int. Lung Cancer Congress. 
Weiner RS (2017) The Tolerance and Safety of 4-Demethyl-4-cholesteryloxypenclomedine [DM-CHOC-PEN] in Adolescent and Young Adult (AYA) Subjects with Advanced Malignancies

12. Sappington DR, Siegel ER, Penney RB, Boysen G (2015) Glutamine, glutaminase and $\mathrm{V}$-glutamyl-transferase activities are essential for lung tumorigenesis. Proc AACR.

13. Mohamed A, Deng X, Khuri FR, Owoniko TK (2014) Altered glutamine metabolism and therapeutic opportunities for lung cancer. Clin Lung Cancer 15: 7-15. [Crossref]

14. Morgan LR, Benes E, Rodgers AH, Weiner RS (2014) Safety and use of DM-CHOCPEN in patients with advanced cancers. Presented to the FDA, Dec. 2014, IND-68,876.
15. Results of the Phase I trial for 4-demethylpenclomedine. Personnel communication, 2013.

16. Toronezos ES (2017) Discussions on adolescent and young adult survivorship. 2017 Cancer Survivor- ship Symposium, 2017.

17. Doyle C (2017) What do we still need to know about adolescent and young adult survivorship? Oncology Practice Management 5: 34-35.

Copyright: $@ 2017$ Weiner RS. This is an open-access article distributed under the terms of the Creative Commons Attribution License, which permits unrestricted use, distribution, and reproduction in any medium, provided the original author and source are credited. 